\title{
Cardiovascular and Cerebrovascular Disease Incidence Among 42785 Adults: The Thai Cohort Study, 2005-2013
}

\author{
Xiyu Feng ${ }^{1}$, Matthew Kelly ${ }^{1}$, Sam-ang Seubsman ${ }^{2} \&$ Adrian Sleigh ${ }^{1}$ \\ ${ }^{1}$ Department of Global Health, Research School of Population Health, the Australian National University, \\ Canberra, Australia \\ ${ }^{2}$ School of Human Ecology, Sukhothai Thammathirat Open University, Nonthaburi, Thailand \\ Correspondence: Matthew Kelly, RSPH, 62 Mills Rd, ANU, Canberra 2602, Australia. Tel: 61-2-6125-0714. \\ E-mail: matthew.kelly@anu.edu.au
}

Received: March 6, 2020 Accepted: April 26, 2020 Online Published: May 10, 2020

doi:10.5539/gjhs.v12n7p81 URL: https://doi.org/10.5539/gjhs.v12n7p81

This study was supported by the International Collaborative Research Grants Scheme with joint grants from the Wellcome Trust UK (GR071587MA) and the Australian National Health and Medical Research Council (268055), and as a global health grant from the NHMRC (585426).

\begin{abstract}
Background: Due to economic and social development in Thailand, cardiovascular disease (CVD) and cerebrovascular disease (CVA) have been gradually replacing infectious diseases and have become the main threat to health in this country.

Method: This study used the 2005 baseline data of 42785 members of the Thai cohort study (TCS) to identify health risk factors correlated with incidence of CVD and/or CVA over 8 years (2005- 2013). We applied multivariate logistic regression to investigate associations between demographic and socioeconomic factors, health conditions, and personal lifestyle factors and CVD and/or CVA incidence.

Results: The cumulative incidence of CVD and/or CVA in males was more than three times that in females. CVD and/or CVA incidence was correlated with ageing, obesity (AOR: 1.67, 95\% CI: 1.16-2.40) and previous diagnosis with diabetes (AOR: 3.09, 95\% CI: 1.80-5.30), hyperlipidaemia (AOR: 1.54, 95\% CI: 1.08-2.19), hypertension (AOR: 1.71, 95\% CI: 1.13-2.59), chronic kidney disease (AOR: 2.35, 95\% CI: 1.35-4.10), and depression/anxiety (AOR: $2.76,95 \%$ CI: 1.64-4.63). Short sleep time was positively associated with CVD and/or CVA in the Thai Cohort Study. An inverse association between performing housework and the incidence of CVD and/or CVA was also identified. However, current smoking had a significant positive correlation with the incidence of CVD and/or CVA for participants.
\end{abstract}

Conclusion: Older age, obesity, underlying diseases, short sleep time, and current smoking were the risk factors for CVD and/or CVA incidence for the participants. However, housework, as an incidental exercise, could protect people against the risk of CVD and/or CVA.

Keywords: cardiovascular disease, cerebrovascular disease, personal lifestyle factors, socio-economic status, Thai Cohort study, underlying disease.

\section{Introduction}

Cardiovascular disease (CVD) and cerebrovascular disorders (CVA) are the ischemic or haemorrhagic disorders of the heart, brain and systemic tissues caused by conditions including hyperlipidaemia, blood viscosity, atherosclerosis, and hypertension (World Health Organization [WHO], 2019a). CVD and CVA are serious threats to human health due to high morbidity and high mortality. CVD and CVA are the leading causes of death globally, and according to the report of the World Health Organisation (WHO), over 13 million CVD and CVA deaths occur in low and middle income countries and regions, accounting for about $75 \%$ of the total global CVD and CVA deaths (WHO, 2019b).

Thailand, as one of the developing countries in Southeast Asia, bears a huge burden of CVD and CVA, just like other middle-income nations around the world (Kaptoge et al., 2019). Stroke and ischaemic heart disease are two cardiovascular and cerebrovascular illnesses with the highest morbidity and mortality. From 2005 to 2017 , the Disability-Adjusted Life Year (DALY) loss caused by stroke increased by about 100,000, while that led by 
ischemic heart disease rose by about 104,000 (Institute for Health Metrics and Evaluation [IHME], 2019). Ischemic heart disease and stroke are now the leading causes of death and cause a large burden for the Thai people. The phenomenon is related to many social-environmental and personal factors. In Southeast Asia, urbanization, low social-economic status (SES), the sedentary lifestyle, effects of smoking and drinking, as well as co-morbidity with diseases such as diabetes, are the health risk factors which in other studies have been associated with CVD and CVA (Roth et al., 2017).

CVD and CVA are non-communicable chronic diseases (NCDs) with high mortality burdens. However, there are limited longitudinal studies in Thailand which can identify long term health risk factors for CVD or CVA incidence. Therefore, from 2005, a cohort study of more than 85,000 Thai people living throughout the country was established, namely, the Thai Cohort Study (TCS). The risks assessed in this study included many aspects such as social, occupational, geographic, and disease history. This study providing valuable information on the life-course health risks for Thailand people, as the country's disease burden is in transition.

The analysis presented here addresses the association between health risk elements and the incidence of CVD and/or CVA in TCS from 2005 to 2013. Our findings will inform the development of prevention and control programs by identifying those most at risk.

\section{Methods}

\subsection{Study Population}

The Thai Cohort Study (TCS) is a nationwide project, investigating the health risk transition underway in the country from the traditional infectious disease model to the emerging non-communicable chronic diseases (NCDs). This project started in 2005 and has recruited 87,151 individuals who were enrolled at Sukhothai Thammathirat Open University (STOU) in Thailand (Sleigh, Seubsman, Bain, \& Thai Cohort Study Team, 2008). Participants in this study came from all over the country and were aged 15-87 years at baseline. These respondents were a reasonable representation of the STOU student body and the general Thai population since their median age, median income, national residence, and ethnic diversity were similar to the national population structure (Sleigh, Seubsman, Bain, \& Thai Cohort Study Team, 2008). Nevertheless, they are younger and more educated than national adult population in Thailand and the proportion of STOU students who were living in urban areas was also relatively higher. Thus, although TCS members may not be representative of the entire Thai population, they can potentially represent the development of the Thai population in the future and they were also in a more advanced position on the path of the transition of health risks (Sleigh, Seubsman, Bain, \& Thai Cohort Study Team, 2008).

Data collection comprised three mail-out questionnaires. The first was in 2005 (baseline), with follow-up surveys in 2009 and 2013. At baseline, questionnaires were sent to all 200,000 students enrolled at Sukhothai Thammathirat Open University (STOU). 87151 responded and these participants from the baseline population for this study. At each round of follow-up, in 2009 and 2013, 70\% of the cohort were retained in the study. This meant that around 40,000 participants completed the final 2013 follow-up. The content of three questionnaires was very rich, including basic demographic information (e.g. age, gender, home address), work and income, living and working environment, personal and family disease history, personal lifestyle factors (e.g. diets, physical activities, and smoking and drinking), injures and transportation, as well as other related information.

\subsection{Incidence of CVD and/or CVA}

At all 3 follow-up rounds a question was asked whether respondents had ever been diagnosed by a doctor with any of a list of 24 diseases. Incident CVD and/or CVA cases were defined by reporting 'no' to having being diagnosed with CVD and/or CVA at baseline in 2005, and 'yes' to these questions in 2013. This is defined as 8-year incidence.

\subsection{Health Risk Factors}

There are many potential risk factors associated with CVD and/or CVA, which could be divided into the following four categories.

The first category was demographic factors. Gender (male and female age (three groups: less than 29 years old, 30-39 years old and over 40 years old), and marital status (married/living with a partner or being single) were included this category. Urbanization status was categorized in terms of rural (R) or urban (U) residential residence, when aged 10-12 years old and again in 2005, creating four groups: lifelong ruralites (RR), urbanizers (RU), de-urbanizers (UR) and urbanites (UU).

The second category was social-economic status (SES). Personal monthly income was classified as five groups: less than 7,000 baht, from 7,001 to 10,000 baht, from 10,001 to 20,000 baht and over 20001 baht. The monthly household income was categorized as three groups: the lower group (less than 30,000 baht), the middle group (from 30,001 to 60,000 baht) and the higher one (over 60,001 baht). One US dollar was equivalent to around 35 baht in 2005. There are three groups of completed educational levels: high school, post-high school diploma, 
university's bachelor degree or above.

The third category was health conditions such as obesity and overweight, diabetes, hyperlipidaemia, hypertension, chronic kidney disease and emotional health problems (depression and anxiety) that could increase the risk of CVD and/or CVA. Obesity and overweight were defined using participants' body mass index (BMI). The Asian criteria of BMI was used in the study: BMI less than 18.5 (underweight), BMI between 18.5 and 23.0 (normal), BMI between 23.0 and 25.0 (overweight), BMI more than 25.0 (obese) (Banks, Lim, Seubsman, Bai, \& Sleigh, 2011).

The fourth category were the personal lifestyle factors variables. The frequency of diet consumption (deep fried food, roasted/smoked food, instant foods, soft drink and soybean products) was categorized into five groups: less than one time per month (1), 1-3 times per month (2), 1-2 times per week (3), 3-6 times per week (4), daily or more (5). Western fast food was classified three groups: less than one time per month (1), less than 3 times per month (2) and over 3 times per month (3). The intake of fruit and vegetables would be noted as standard serves each day.

As for exercise per week, there are four groups, namely, "strenuous exercise for more than 20 minutes", "moderate exercise for more than 20 minutes", "mild exercise for more than 20 minutes" and "walking for at least 10 minutes". We calculated in the adjusted exercise measure weighting to evaluate the weekly physical activities: " $2 \times$ vigorous exercises $+1 \times$ moderate exercises $+1 \times$ walking sessions per week", which was based on the International Physical Activity Questionnaire and the Active Australia Survey methodology (Banks et al., 2011; Thawornchaisit et al., 2013; Zhao et al., 2015). Housework was measured as another form of physical activity. The frequency of this was divided into 5 groups: never, 1-3 times per month, 1-2 times per week, 3-4 times per week and most days. The length of time of watching TV and/or computer and sitting for any purpose (the number of hours per day) was analysed in this study to evaluate sedentariness. The length of time of sleeping (the number of hours per day) was also measured.

Smoking status was measured and categorized into three subgroups: "never", "ex-smoker", or "current smoker". As for alcohol drinking status, there were four categories: "never", "ex-drinker", "occasional social drinker" and "current regular drinker".

\subsection{Statistical Analysis}

All analyses were performed using Stata 14.0. The 8-year CVD and/or CVA incidence and its 95\% CI were computed separately for male and female participants. Differences between CVD and/or CVA incidence for risk factor groups were assessed using the $\chi^{2}$ test. Statistical significance was defined as $p$ value less than $5 \%$.

Logistic regression was used to analyse the association between the health risk factors and the incidence of CVD and/or CVA from 2005 to 2013. Due to the stratified analysis of gender, the first step was to use bivariate logistic regression to analyse the association between health risk factors measured at baseline and CVD and/or CVA incidence over the following 8 years. Multivariate logistic regression models were used to assess the multivariate adjusted odd ratios (ORs). For each set of associations, we adjusted for a different set of confounders based on observations on our descriptive data and from other literatures. The details were included on the Suppl. Table 1.

\subsection{Ethical Considerations}

Ethical approval was provided by the Sukhothai Thammathirat Open University Research and Development Institute and Human Research Ethics Committee of the Australian National University.

\section{Results}

\subsection{The Baseline Characteristics of the Study}

Table 1 describes the baseline characteristics of the Thai cohort study in 2005. It was clear that there were more women participants than the men. In terms of the marital status, the number of singles and cohabitants was similar, accounting for $49.8 \%$ and $50.2 \%$ of the total participants respectively. The ratio of single women was higher than that of men. Moreover, the female educational attainment (31.4\% at university level) was higher than males $(28.2 \%$ at university level) in 2005. However, as for the personal salary, around $16.5 \%$ of men earned over 20000 baht in one month while only less than $10 \%$ of women reached this level.

\subsection{The Cumulative Incidence of CVD and/or CVA}

As for diagnosed CVD and/or CVA in the Thai Cohort Study in 2013 (Table 2), the cumulative incidence of CVD and/or CVA among the whole participants was $0.52 \%$ and the incidence in males was more three times than that in females $(0.84 \%$ vs. $0.26 \%$; see the Table 2.). In men ( $\mathrm{p}$-trend $=0.008)$ and women ( $\mathrm{p}$-trend $=0.0196)$ as well as the total study population ( $\mathrm{p}$-trend $=0.0146$ ), the incidence of such diseases increased with age. Furthermore, people living with a partner (male: $1.02 \%$; female: $0.36 \%$ ) were more susceptible to CVD and/or CVA than single persons (male:0.48\%; female: $0.19 \%$ ) in both genders. It was worth noting that participants who moved from the urban area to the countryside (UR) were more likely to suffer from these diseases (RR: $0.84 \%$ vs. RU: $0.58 \%$ vs. UU: $0.63 \%$ 
vs. UR:1.13\%). Moreover, participants with monthly incomes exceeding 20,000 baht had the highest incidence of CVD and/or CVA, compared to other income groups $(<=7000$ baht: $0.37 \%$ vs. $7001-10000$ baht: $0.27 \%$ vs. 10001-20000 baht: $0.66 \%$ vs. $<=20001$ baht: $1.05 \%$ ). People who were at the middle monthly home income level had the highest incidence of CVD and/or CVA (low: $0.35 \%$ vs. middle: $0.68 \%$ vs. high: $0.57 \%$ ). The total participants who had completed a university degree had the lowest incidence of these illnesses (high school: $0.54 \%$ vs. diploma: $0.49 \%$; vs. university: $0.45 \%$ ).

For the total members in this study, the participants who suffer from obesity $(1.21 \%$ vs. $0.22 \%)$, diabetes $(4.93 \%$ vs. $0.47 \%)$, hyperlipidaemia $(1.45 \%$ vs. $0.41 \%)$, hypertension $(2.02 \%$ vs. $0.44 \%)$, chronic kidney disease $(1.57 \%$ vs. $0.49 \%$ ) and depression/anxiety (1.53\% vs. $0.49 \%$ ) had higher incidence of CVD and/or CVA than others (Table 2.). People with the sleep time of 7 to 8 hours per day had the lowest incidence of such diseases $(<=6$ hours: $0.67 \% \mathrm{vs}$. 7-8 hours: $0.45 \%$ vs. $>=9$ hours: $0.46 \%$ ). It was interesting that both male ( $<=7$ hours: $0.96 \%$ vs. $8-12$ hours: $0.71 \%$ vs. $>=13$ hours: $0.78 \%$ ) and female ( $<=7$ hours: $0.34 \%$ vs. $8-12$ hours: $0.27 \%$ vs. $>=13$ hours: $0.18 \%$ ) participants who had sedentary time less than 7 hours per day had the highest incidence of CVD and/or CVA (see the Suppl. Table 2.1.).

The incidence of the diseases of interest was highest in people who consumed smoked food once or more times per day $(0.82 \%)$. In addition, drinking soft drinks once or several times per day also increased incidence of CVD and/or CVA among the total participants (0.67\%) (see the Suppl. Table 2.2.). However, the more vegetables the person ate, the lower incidence of CVD and/or CVA for this individual (p-trend=0.0460). Moreover, the incidence of these diseases was also highest in people who smoked (current smoker vs. never:1.23\% vs. $0.33 \%$ ) or drank alcohol regularly (current regular vs. never: $1.19 \%$ vs. 0.37\%) (Table 2.).

\subsection{The Association between Risk Factors and CVD and/or CVA among Participants}

There was a linear positive relationship between age and CVD and/or CVA in males (p-trend=0.0307) and total participants $(p$-trend $=0.028$ ) after controlling for other variables (Table 3.). However, after controlling for variables including age, marital status and other disease diagnoses, there was no association between personal monthly income and CVD and/or CVA in the total members of the study. Additionally, monthly home income was not be a health risk factor of CVD and/or CVA in men or women separately.

Among all participants, obese individuals had a higher likelihood of CVD and/or CVA incidence when compared with those of normal body size (AOR: 1.67, 95\% CI: 1.16-2.40). In addition, the risk of CVD and/or CVA increased in all the participants who had diabetes (AOR: 3.09, 95\% CI: 1.80-5.30), hyperlipidaemia (AOR: 1.54, 95\% CI: 1.08-2.19), hypertension (AOR: 1.71, 95\% CI: 1.13-2.59), chronic kidney disease (AOR: 2.35, 95\% CI: 1.35-4.10) and depression/anxiety (AOR: 2.76, 95\% CI: 1.64-4.63) (Table 3.). However, most of the personal lifestyle factors such as physical activities and food intake habits (deep-fried food, instant food, soft drink, roasted/smoked food, soybean products, western food, vegetables, and fruits) did not have significant relationships with CVD and/or CVA (see the Suppl. Table 3.1. and the Suppl. Table 3.2.).

However, it was noteworthy that participants whose sleeping time averaged less than 6 hours had positive associations with CVD and/or CVA (AOR: 1.47, 95\% CI: 1.07-2.01) after adjusting for other personal lifestyle elements, socio-demographic factors and health conditions. Moreover, housework (household cleaning or gardening work), one type of physical activity, was a protective factor against such diseases (Table 3).

It was obvious that current smoking increased by about 1.58 times the odds of catching CVD and/or CVA compared to those who did not smoke. Conversely, alcohol consumption had little remaining effect on these diseases (Table 3).

\section{Discussion}

Following economic and social development in Thailand, health problems for the population are gradually transferring from infectious diseases to chronic diseases (Khatab et al., 2019; Sleigh, Seubsman, Bain, \& Thai Cohort Study Team, 2008; Thawornchaisit et al., 2013; Zhao et al., 2015). CVD and CVA are now common in this country with increasing incidence every year (Banks et al., 2011). This increased incidence is potentially being driven by many factors. We studied the relationship between CVD and/or CVA and risk factors and found that age, obesity, diabetes, hyperlipidemia, hypertension, chronic kidney disease, short sleep time, smoking and mental illnesses were closely related to the incidence of CVD and/or CVA. However, housework, as a form of incidental exercise, could protect people against CVD and/or CVA.

\subsection{Underlying Diseases}

Obesity is recognized as one of the major causes of CVD and CVA. The result of the study is consistent with the other longitudinal studies in Asia, which have reported that in obese patients the risk of incident CVD rose by from $27 \%$ to $97 \%$. Obesity can directly affect the heart, causing ventricular hypertrophy and diastolic dysfunction. Moreover, obesity can also induce diabetes, high blood lipids and high blood pressure (Chen et al., 2019). 
These existing chronic conditions, as well as kidney disease are also associated with the development of CVD and CVA in this Thai population. Similar cohort studies among Asian populations have also reported these relationships (Ueshima et al., 2008; Wu et al., 2015). All of these conditions have vascular and cardiovascular effects which can increase CVD/CVA risk.

Renal failure in particular can affect heart function, leading to ischemic heart disease and possibly leading to stroke (Dad \& Weiner, 2015). The result is supported by a cohort study in the USA (adjusted risk ratio (ARR): 2.3, 95\% CI:1.2-3.4) (Bansal et al., 2017). Moreover, our results are also consistent with the systematic review of a cohort study of chronic kidney disease and the risk of stroke (adjusted risk ratio (ARR): 2.18, 95\% CI: 1.68-2.84) (Masson et al., 2015).

The relationship between depression and CVD/CVA in this study is also worth noting. The American Heart Association suggests that depression or anxiety should be considered a major risk factor for coronary heart disease (Chaddha, Robinson, Kline-Rogers, Alexandris-Souphis, \& Rubenfire, 2016). This may be because the prevalence of CVD/CVA risk factors such as smoking and alcohol consumption is higher in depressed patients (Dhar \& Barton, 2016). Nevertheless, in our study, after controlling for smoking and drinking, mental illnesses are still positively associated with such diseases. This may be due to changes in neurological and endocrine function (Chaddha et al., 2016), however, the association between mental disorders and CVD and CVA is still under investigation.

\subsection{Personal Lifestyle Factors}

We found that current smoking was significantly associated with increased risk of developing CVD and/or CVA in male participants, which is consistent with much other research (Messner \& Bernhard, 2014; Tan et al., 2018).

However, no association was found between alcohol consumption and diagnosed CVD and/or CVA. This may be due to the small number of people diagnosed with CVD and CVA, resulting in insufficient statistical power to detect relationships. However, in global longitudinal studies, alcohol has been shown to increase the risk of CVD and CVA development, namely, after controlling for other risk factors including age, SES, family disease history, and smoking, the larger amount of alcohol a person intakes, the higher the risk of stroke and coronary heart disease the individual gets (Wood et al., 2018).

Short sleep time was positively associated with CVD and/or CVA in the Thai cohort study. Furthermore, it is worth noting that people with sleep time less than 7 hours or more than 8 hours have a higher incidence of CVD and/or CVA than normal duration of sleeping times, which is consistent with a cross-sectional study in the USA (Buxton \& Marcelli, 2010). Sleeping too much or too little could increase the probability of suffering from CVD and CVA (Buxton \& Marcelli, 2010). However, the cause of these phenomena warrants further study.

Sedentary time did not have any effect on CVD and/or CVA in this cohort study. However, physical activity in the form of housework protected participants against CVD and/or CVA; no relationship was found with purposeful physical activity. Housework reducing the risk of CVD and/or CVA is consistent with other research among Asian Indian populations (Nag \& Ghosh, 2013). Exercise may improve the function of blood vessels and inhibit arteriosclerosis, thereby reducing the risk of these illnesses (Nystoriak \& Bhatnagar, 2018).

\subsection{Socio-Demographic Factors}

We also note that the influence of age on the incidence of such diseases is consistent with the results of many similar studies. For example, a cross-sectional study in Thailand (2008-2009) showed that the older age of people was, the higher the risk of stroke and coronary heart disease (Khatab et al., 2019). In TCS, the cumulative incidence of CVD and/or CVA in males was more three times than that in females, which is similar with the worldwide CVD study that found men in Southeast Asia are at higher risk of developing such diseases than women (Roth et al., 2017). It is a known factor that males' incidence of CVD and CVA is higher than females. Nevertheless, as TCS participants may not represent the total Thai population, the results found here will not reflect information about the incidence of these diseases in males being higher than that in females nationally (Roth et al., 2017; Thawornchaisit et al., 2013; Thawornchaisit et al., 2015).

In many studies in various settings socio-economic status in terms of income education and assets are associated with CVD and CVA risk. This relationship was not found in our cohort study. This may be due to the fact that our participants were all Open University students and displayed less extremes of high and low socio-economic status. As well, socio-economic status indicators are also often proxies for differences in biological and personal lifestyle risk factors, for example, higher income TCS members also have a higher average age.

However, low-SES is often associated with more risk taking behavior in terms of smoking and alcohol and also poorer diets, which increases the risk of coronary heart disease and stroke, compared with other people (Psaltopoulou et al., 2017). 


\section{Strengths and Limitations}

In this study, we found the relationship between risk factors and diagnosed CVD and CVA. The TCS is a relatively mature and comprehensive study of health transitions in Thailand. This is the first study of the relationship between CVD and CVA and related factors from 2005 to 2013 in TCS.

Although the sample size of this study is large, the population coverage is relatively wide, and the research duration is relatively long, there are still some limitations in our study.

Firstly, our participants were all open university students at baseline, which means that they may not be able to represent the entire Thai population. Also, the participants are younger and more educated and may not manifest CVD and CVA until later in life. This means that incidence may be lower in the TCS than the general population. Thus, TCS participants may thus represent the next generation of people in Thailand (Thawornchaisit et al., 2013; Thawornchaisit et al., 2015). As well, despite the cohort population not being representative of the Thai national population, the associations found between risk factors and disease outcomes in the cohort are still valid and applicable. Moreover, in this 8-year study, we inferred the risk factors associated with CVD and CVA based on the characteristics of the participants in the baseline (2005), which may cause some bias because participants may change their habits within 8 years, resulting in inaccurate results. Furthermore, this study relies on self-reporting, which may lead to recall bias. This kind of bias belong to systematic error, and it is impossible to eliminate the error by increasing the sample size, thus it may have a certain impact on the results presented here.

\section{Conclusion}

In summary, older age, obesity and some disease factors such as hyperlipidemia, hypertension, diabetes, chronic kidney disease, and depression/anxiety were closely related to increased incidence of CVD and/or CVA in men and women. There was a positive relationship between short sleep time and CVD and/or CVA in the Thai cohort study. Although the purposeful physical activities had less effect on these diseases, housework had an inverse correlation with the incidence of CVD and/or CVA among the participants. However, current smoking is still the main risk factors for incidence of CVD and/or CVA.

\section{Recommendations}

In addition to controlling smoking and drinking, the Thai government should devote attention to those diagnosed with mental illnesses such as depression/anxiety and other chronic co-morbidities to help relieve the burden of CVD and CVA in the Thai population. Currently, Thailand has a relatively complete diagnostic system for depression (Sharan, Sagar, \& Kumar, 2017). Furthermore, the authorities should also advocate that the incidental house and garden work type exercise as just as beneficial as intentional exercise, and urge people to actively participate in physical exercise, thereby reducing the incidence of CVD and CVA.

\section{Acknowledgements}

We thank the staff at the Sukhothai Thammathirat Open University (STOU) who assisted with student contact, and the STOU students who are participating in the cohort study. We also thank the Thai Ministry of Interior for mortality data linkage.

\section{Competing Interests Statement}

All authors declare that they have no competing interests.

\section{References}

Banks, E., Lim, L., Seubsman, S., Bain, C., \& Sleigh, A. (2011). Relationship of obesity to physical activity, domestic activities, and sedentary behaviours: Cross-sectional findings from a national cohort of over 70,000 Thai adults. BMC Public Health, 11(1), 762-775. https://doi.org/10.1186/1471-2458-11-762

Bansal, N., Katz, R., Robinson-Cohen, C., Odden, M. C., Dalrymple, L., \& Shlipak, M. G. (2017). Absolute rates of heart failure, coronary heart disease, and stroke in chronic kidney disease: An Analysis of 3 community-based cohort studies. JAMA Cardiology, 2(3), 314-318. https://doi.org/10.1001/jamacardio.2016.4652

Buxton, O. M., \& Marcelli, E. (2010). Short and long sleep are positively associated with obesity, diabetes, hypertension, and cardiovascular disease among adults in the united states. Social Science \& Medicine, 71(5), 1027-1036. https://doi.org/10.1016/j.socscimed.2010.05.041

Chaddha, A., Robinson, Elizabeth A., Kline-Rogers, E., Alexandris-Souphis, T., \& Rubenfire, M., (2016). Mental health and cardiovascular disease. American Journal of Medicine, 129(11), 1145-1148. https://doi.org/10.1016/j.amjmed.2016.05.018

Chen, Y., Copeland, W. K., Vedanthan, R., Grant, E., Lee, J. E., Gu, D., ... Potter, J. D. (2013). Association between body mass index and cardiovascular disease mortality in East Asians and South Asians: Pooled analysis of 
prospective data from the Asia Cohort Consortium. BMJ: British Medical Journal, 347(7927), 12-30. https://doi.org/10.1136/bmj.f5446

Dad, T., \& Weiner, D. E. (2015). Stroke and chronic kidney disease: Epidemiology, pathogenesis, and management across kidney disease stages. Seminars in Nephrology, 35(4), 311-322. https://doi.org/10.1016/j.semnephrol.2015.06.003

Dhar, A. K., \& Barton, D. A. (2016). Depression and the link with cardiovascular disease. Frontiers in Psychiatry, 7, 33-41. https://doi.org/10.3389/fpsyt.2016.00033

Institute for Health Metrics and Evaluation. (2019). GBD Results Tool. Retrieved from http://ghdx.healthdata.org/gbd-results-tool

Kaptoge, S., Pennells, L., De Bacquer, D., Cooney, M. T., Kavousi, M., ... Angelantonio, E. D. (2019). World health organization cardiovascular disease risk charts: Revised models to estimate risk in 21 global regions. The Lancet Global Health, 7(10), e1332-e1345. https://doi.org/10.1016/S2214-109X(19)30318-3

Khatab, K., Inthawong, R., Whitfield, M., Collins, K., Raheen, M., \& Ismail, M. Risk factors associated with cardiovascular disease (CVD) in Thailand from the 4th National Health Examination Survey 2008-2009. (2019). Biostatistics and Epidemiology International Journal, 2(1), 10-19. https://doi.org/10.30881/beij.00014

Masson, P., Webster, A. C., Hong, M., Turner, R., Lindley, R. I., \& Craig, J. C. (2015). Chronic kidney disease and the risk of stroke: A systematic review and meta-analysis. Nephrology Dialysis Transplantation, 30(7), 1162-1169. https://doi.org/10.1093/ndt/gfv009

Messner, B., \& Bernhard, D. (2014). Smoking and cardiovascular disease: Mechanisms of endothelial dysfunction and early atherogenesis. Arteriosclerosis, Thrombosis, and Vascular Biology, 34(3), 509-515. https://doi.org/10.1161/ATVBAHA.113.300156.

Nag, T., \& Ghosh, A. (2013). Cardiovascular disease risk factors in Asian Indian population: A systematic review. Journal of Cardiovascular Disease Research, 4(4), 222-228. https://doi.org/10.1016/j.jcdr.2014.01.004

Nystoriak, M. A., \& Bhatnagar, A. (2018). Cardiovascular effects and benefits of exercise. Frontiers in Cardiovascular Medicine, 5, 135-145. https://doi.org/10.3389/fcvm.2018.00135

Psaltopoulou, T., Hatzis, G., Papageorgiou, N., Androulakis, E., Briasoulis, A., \& Tousoulis, D. (2017). Socioeconomic status and risk factors for cardiovascular disease: Impact of dietary mediators. Hellenic Journal of Cardiology, 58(1), 32-42. https://doi.org/10.1016/j.hjc.2017.01.022

Roth, G. A., Johnson, C., Abajobir, A., Abd-Allah, F., Abera, S. F., ... Murray, C. (2017). Global, regional, and national burden of cardiovascular diseases for 10 causes, 1990 to 2015. Journal of the American College of Cardiology, 70(1), 1-25. https://doi.org/10.1016/j.jacc.2017.04.052

Sharan, P., Sagar, R., \& Kumar, S. (2017). Mental health policies in South-East Asia and the public health role of screening instruments for depression. WHO South-East Asia Journal of Public Health, 6(1), 5-11. https://doi.org/10.4103/2224-3151.206165

Sleigh, A. C., Seubsman, S., Bain, C., \& the Thai Cohort Study Team. (2008). Cohort profile: The Thai Cohort of 87134 Open University students. International Journal of Epidemiology, 37(2), 266-272. https://doi.org/10.1093/ije/dym161

Tan, J., Zhang, X., Wang, W., Yin, P., Guo, X., \& Zhou, M. (2018). Smoking, blood pressure, and cardiovascular disease mortality in a large cohort of Chinese men with 15 years follow-up. International Journal of Environmental Research and Public Health, 15(5), 1026-1034. https://doi.org/10.3390/ijerph15051026

Thawornchaisit, P., de Looze, F., Reid, C. M., Seubsman, S., Sleigh, A. C., \& Thai Cohort Study Team. (2013). Health risk factors and the incidence of hypertension: 4-year prospective findings from a national cohort of 60 569 Thai Open University students. BMJ Open, 3(6), 1-10. https://doi.org/10.1136/bmjopen-2013-002826

Thawornchaisit, P., de Looze, F., Reid, C. M., Seubsman, S., Tran, T. T., Sleigh, A., \& Thai Cohort Study Team. (2015). Health-risk factors and the prevalence of chronic kidney disease: Cross-sectional findings from a national cohort of 87,143 Thai Open University students. Global Journal of Health Science, 7(5), 59-72. https://doi.org/10.5539/gjhs.v7n5p59

Ueshima, H., Sekikawa, A., Miura, K., Turin, T. C., Takashima, N., Kita, Y., ...Okamura, T. (2008). Cardiovascular disease and risk factors in Asia: A selected review. Circulation, 2118(25), 2702-2709. https://doi.org/10.1161/CIRCULATIONAHA.108.790048

Wood, A. M., Kaptoge, S., Butterworth, A. S, Butterworth, A., Willeit, P., Bolton, T., ... Danesh, J. (2018). Risk 
thresholds for alcohol consumption: Combined analysis of individual-participant data for 599912 current drinkers in 83 prospective studies. The Lancet, 391(10129), 1513-1523. https://doi.org/10.1016/S0140-6736(18)30134-X

World Health Organization. (2019a). About cardiovascular diseases. Retrieved from https://www.who.int/cardiovascular_diseases/about_cvd/en/

World Health Organization. (2019b). Cardiovascular diseases (CVDs). Retrieved from https://www.who.int/news-room/fact-sheets/detail/cardiovascular-diseases-(cvds)

Wu, C., Hu, H., Chou, Y., Huang, N., Chou, Y., \& Li, C. (2015). High blood pressure and all-cause and cardiovascular disease mortalities in community-dwelling older adults. Medicine, 94(47), e2160-e2169. https://doi.org/10.1097/MD.0000000000002160

Zhao, J., Kelly, M., Bain, C., Seubsman, S., Sleigh, A., \& Thai Cohort Study Team. (2015). Risk factors for cardiovascular disease mortality among 86866 members of the Thai Cohort Study, 2005-2010. Global Journal of Health Science, 7(1), 107-114. https://doi.org/10.5539/gjhs.v7n1p107

Table 1. The Characteristics of Participants at the Baseline of 2005 Thai Cohort Study

\begin{tabular}{|c|c|c|c|c|c|c|c|}
\hline \multirow{3}{*}{$\begin{array}{l}\text { Variables } \\
\text { Participants }\end{array}$} & \multicolumn{2}{|l|}{ Male } & \multicolumn{2}{|c|}{ Female } & \multirow[t]{2}{*}{ Difference p-value } & \multicolumn{2}{|l|}{ Total } \\
\hline & \multicolumn{2}{|c|}{ N Percent (\%) } & \multicolumn{2}{|c|}{ N Percent (\%) } & & \multicolumn{2}{|c|}{ N Percent (\%) } \\
\hline & 19330 & 45.2 & 23445 & 54.8 & $p<0.001$ & 42785 & 100 \\
\hline Age (years) means (SD) & \multicolumn{2}{|c|}{$34.3(8.87)$} & \multicolumn{2}{|c|}{$31.0(7.83)$} & & \multicolumn{2}{|c|}{$32.5(8.48)$} \\
\hline \multicolumn{8}{|l|}{ Age groups (years) } \\
\hline$<=\mathbf{3 0}$ & 6636 & 34.3 & 11725 & 50.0 & & 18301 & 42.8 \\
\hline $31-40$ & 7453 & 38.6 & 8123 & 34.6 & & 15576 & 36.5 \\
\hline$>=40$ & 5241 & 27.1 & 3607 & 15.4 & & 8848 & 20.7 \\
\hline
\end{tabular}

\begin{tabular}{lcccccc}
\hline Marry status & \multicolumn{1}{l}{} & & & \\
\hline Living with partner & 11259 & 60.0 & 9695 & 42.4 & 20954 & 50.2 \\
\hline Single & 7585 & 40.0 & 13171 & 57.6 & 20756 & 49.8 \\
\hline & $\mathrm{p}<0.001$ & & & & & \\
\hline
\end{tabular}

\begin{tabular}{lcccccc}
\hline Regions & & & & & & \\
\hline Bangkok & 2668 & 13.8 & 4301 & 18.4 & 6969 & 16.4 \\
\hline Central & 4290 & 22.3 & 6891 & 25.2 & 10181 & 23.9 \\
\hline North & 4128 & 21.7 & 4440 & 19.0 & 8568 & 20.1 \\
\hline Northeast & 4746 & 24.7 & 4287 & 18.4 & 9033 & 21.3 \\
\hline East & 1103 & 5.7 & 1367 & 5.9 & 2470 & 5.8 \\
\hline South & 2273 & 11.8 & 3057 & 13.1 & 5330 & 12.5 \\
\hline
\end{tabular}

\begin{tabular}{llllllll}
\multicolumn{9}{c}{$\mathrm{p}<0.001$} & & & & \\
\hline Urbanization status & & & & & 2641 & 18.9 \\
\hline Rural-rural (RR) & 1424 & 20.6 & 1217 & 17.4 & 3971 & 28.6 \\
\hline Rural-urban (RU) & 1921 & 27.8 & 2050 & 29.3 & 4807 & 34.6 \\
\hline Urban-rural (UR) & 2320 & 33.6 & 2487 & 35.5 & 2489 & 17.9 \\
\hline Urban-urban (UU) & 1244 & 18.0 & 1245 & 17.8 & & & \\
\hline
\end{tabular}

\begin{tabular}{lllllll}
\hline Socioeconomic status & & & & \\
\hline Education level & & & & & & \\
\hline High school & 8914 & 48.4 & 8680 & 37.8 & 17594 & 42.5 \\
\hline
\end{tabular}




\begin{tabular}{|c|c|c|c|c|c|c|}
\hline Diploma & 4293 & 23.4 & 7091 & 30.8 & 11384 & 27.5 \\
\hline University & 5177 & 28.2 & 7203 & 31.4 & 12380 & 30.0 \\
\hline \multicolumn{7}{|c|}{$\mathrm{p}<0.001$} \\
\hline \multicolumn{7}{|c|}{ Personal monthly income (baht) } \\
\hline$<=7000$ & 5535 & 29.1 & 9609 & 41.9 & 15144 & 36.1 \\
\hline 7001-10 000 & 4192 & 22.0 & 5397 & 23.5 & 9589 & 22.9 \\
\hline $10001-20000$ & 6163 & 32.4 & 5694 & 24.8 & 11857 & 28.2 \\
\hline$>=20001$ & 3135 & 16.5 & 2235 & 9.8 & 5370 & 12.8 \\
\hline \multicolumn{7}{|c|}{$\mathrm{p}<0.001$} \\
\hline \multicolumn{7}{|l|}{ Household assets (baht) } \\
\hline Means (SD) & \multicolumn{2}{|c|}{$48656(23643)$} & \multicolumn{2}{|c|}{$48701(23626)$} & \multicolumn{2}{|c|}{$48680(23634)$} \\
\hline \multicolumn{7}{|c|}{ Household assets level (baht) } \\
\hline $\operatorname{Low}(<=30,000)$ & 7019 & 36.5 & 8753 & 37.5 & 15772 & 37.0 \\
\hline Medium $(30,001-60,000)$ & 6265 & 32.5 & 7293 & 31.2 & 13558 & 31.8 \\
\hline High $(>=60,001)$ & 5971 & 31.0 & 7308 & 31.3 & 13279 & 31.2 \\
\hline \multicolumn{7}{|c|}{$\mathrm{p}=0.011$} \\
\hline
\end{tabular}




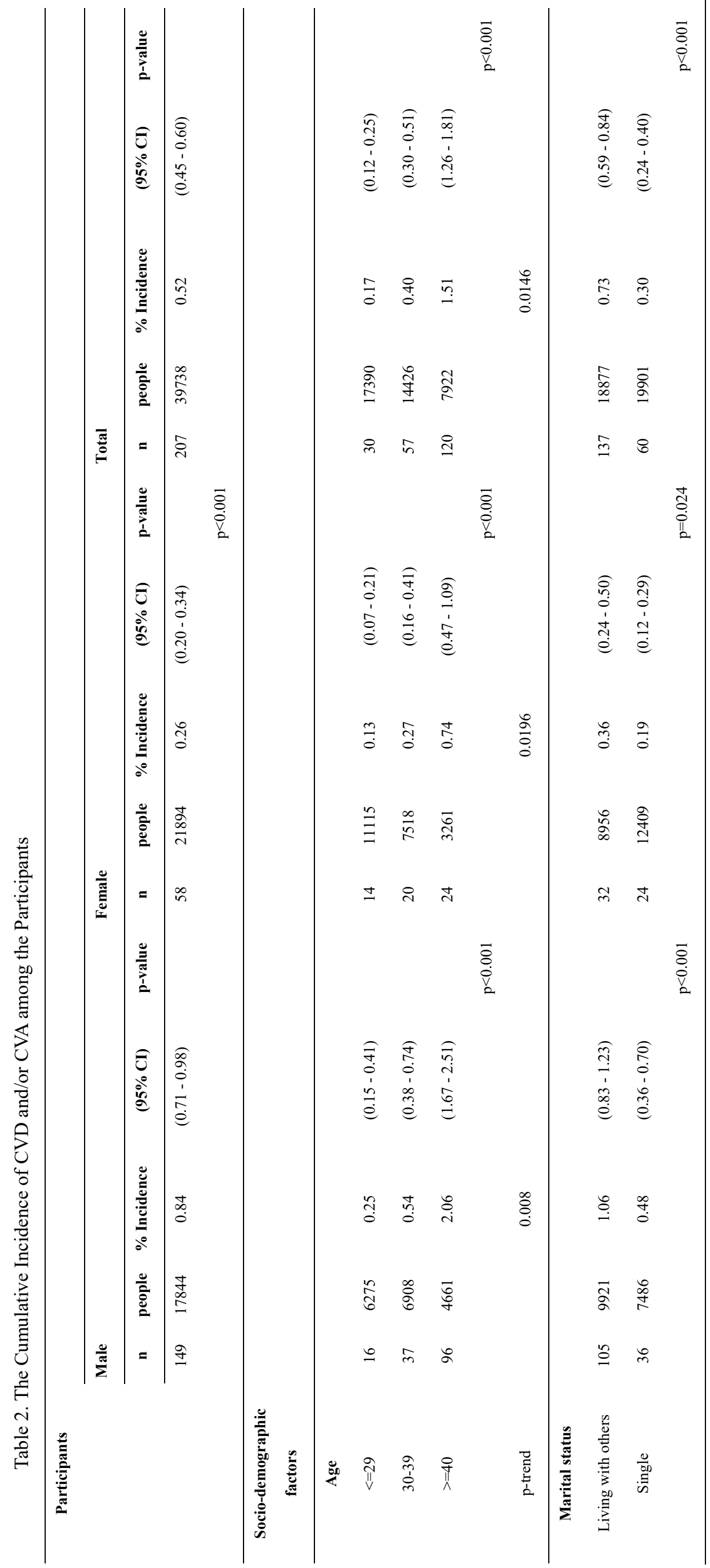




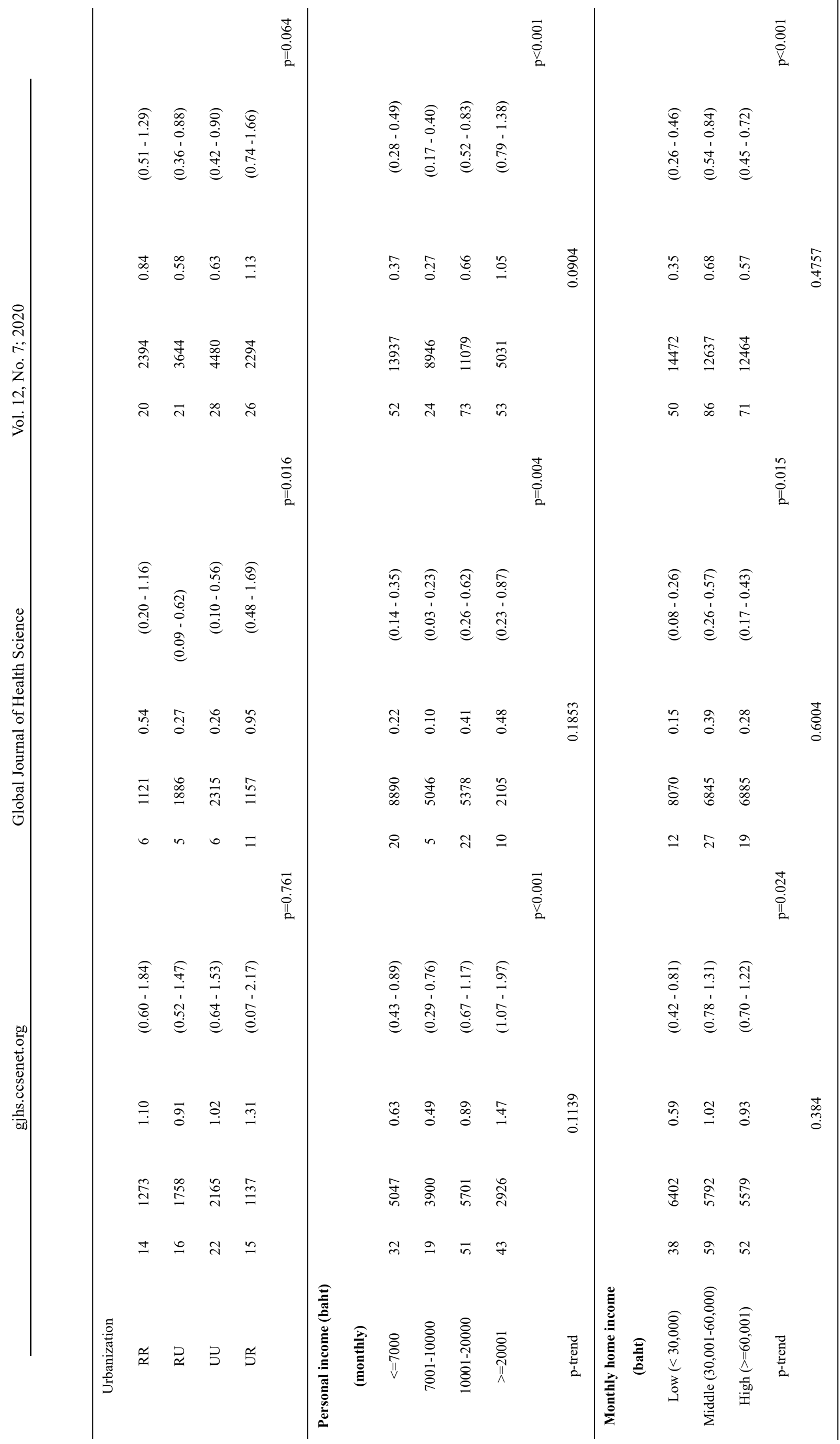




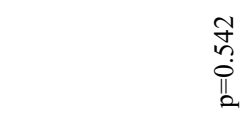

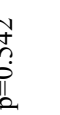

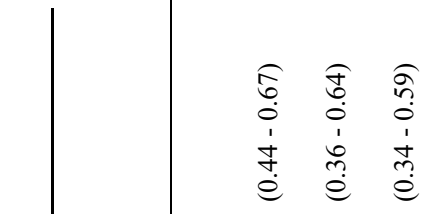

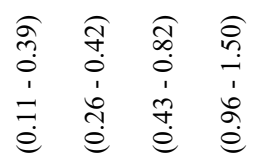

$\bar{\Xi}$
$\dot{0}$
$\stackrel{v}{a}$

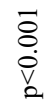

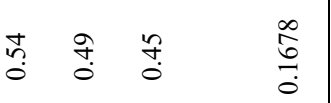

สุ

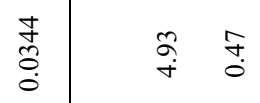

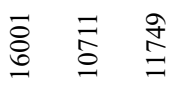

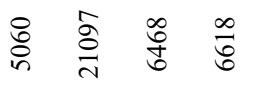

+

$\infty$ in in

$=$ ह के \&

ส

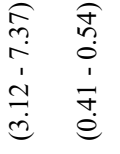

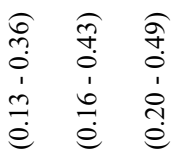

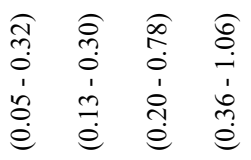

สิ สิ

$\stackrel{\dot{0}}{\stackrel{\infty}{=}}$

กู่

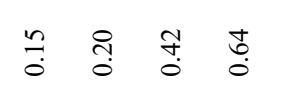

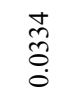

ì d

๙ิ

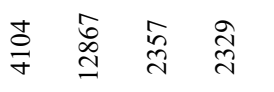

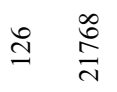

$\simeq \stackrel{2}{\simeq}$

- 는

in in

ڤ্ণ

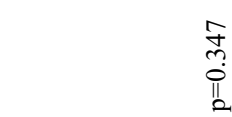

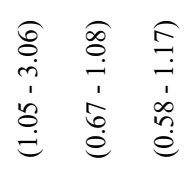

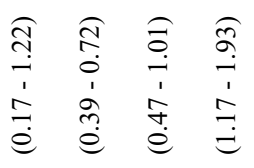

$\underset{\infty}{\infty} \underset{\infty}{\infty} \underset{0}{0}$

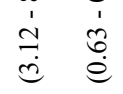

索

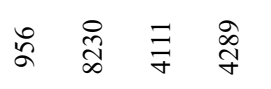

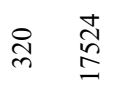

8. मे

n ₹ ते ह

$=\cong$

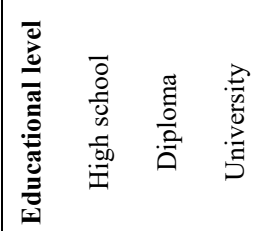

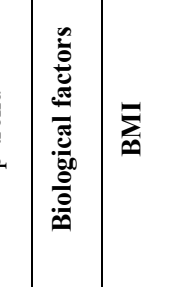

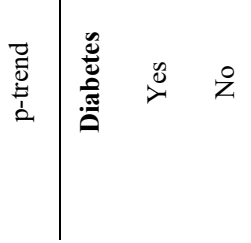




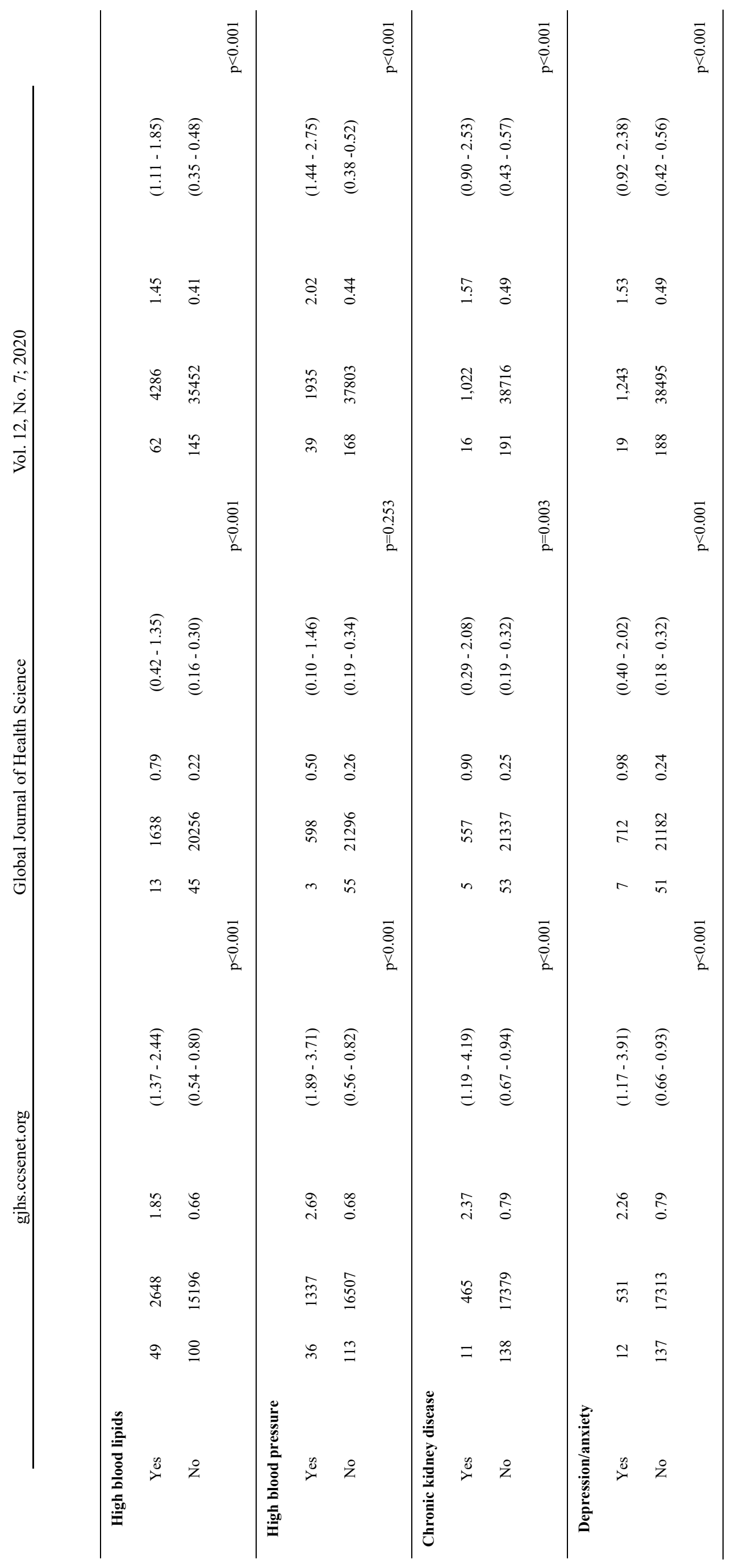




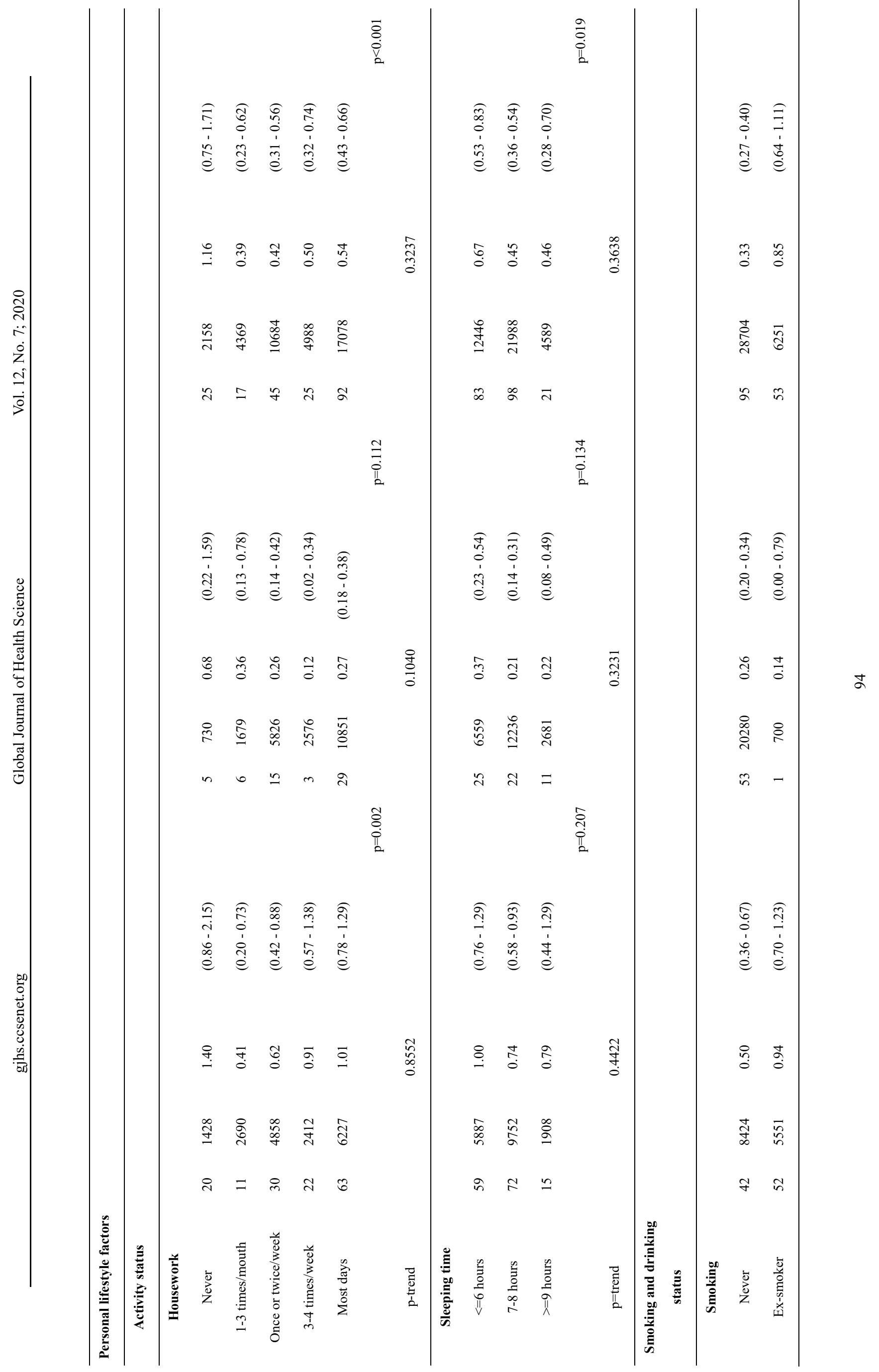




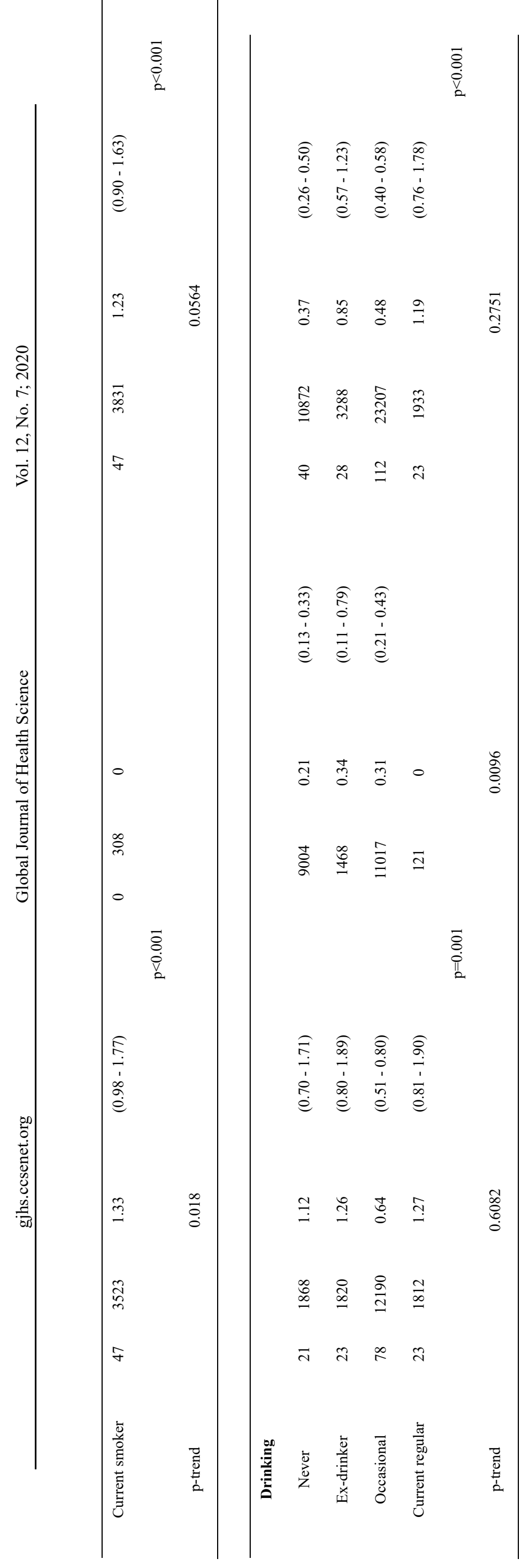




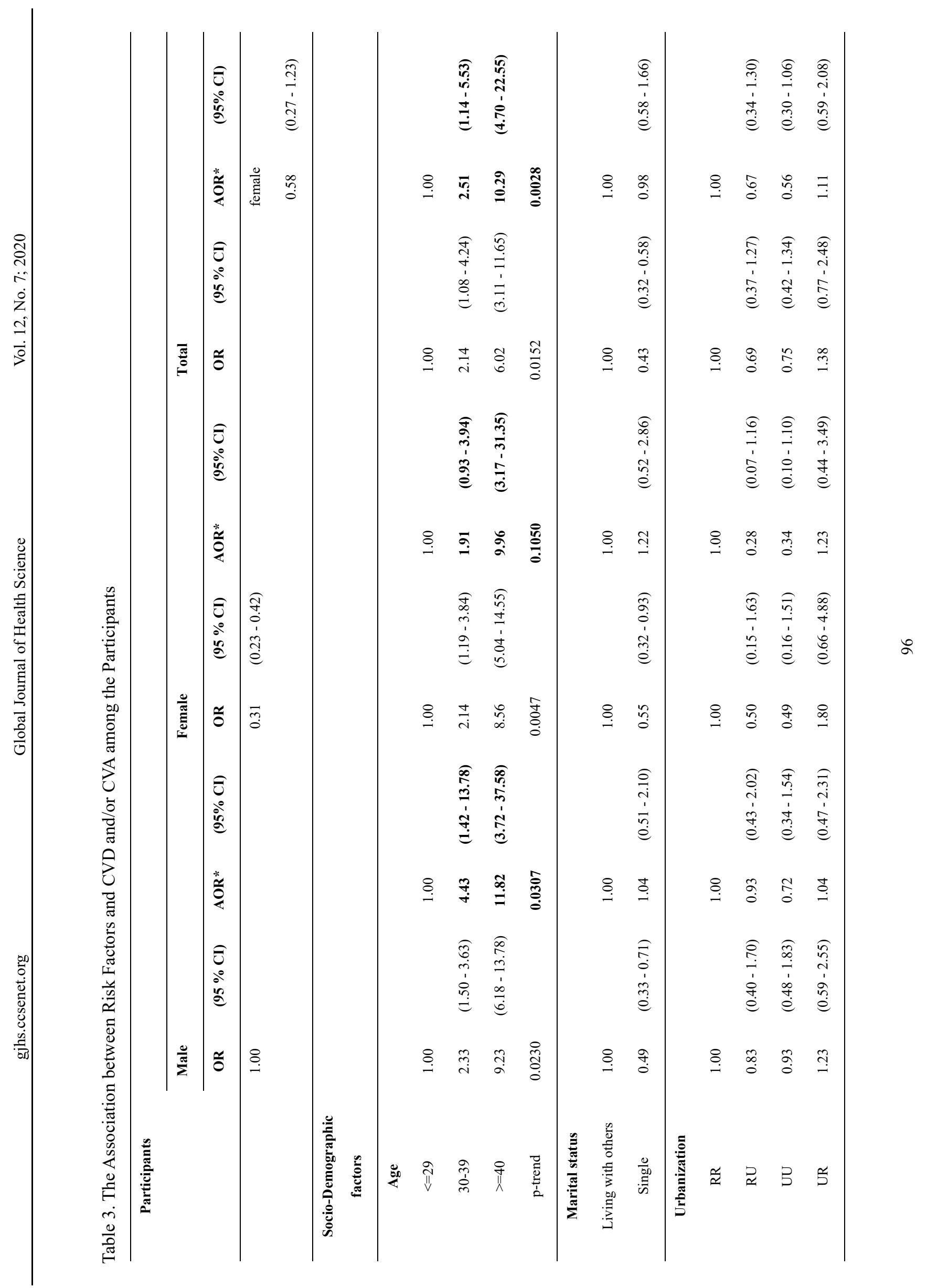




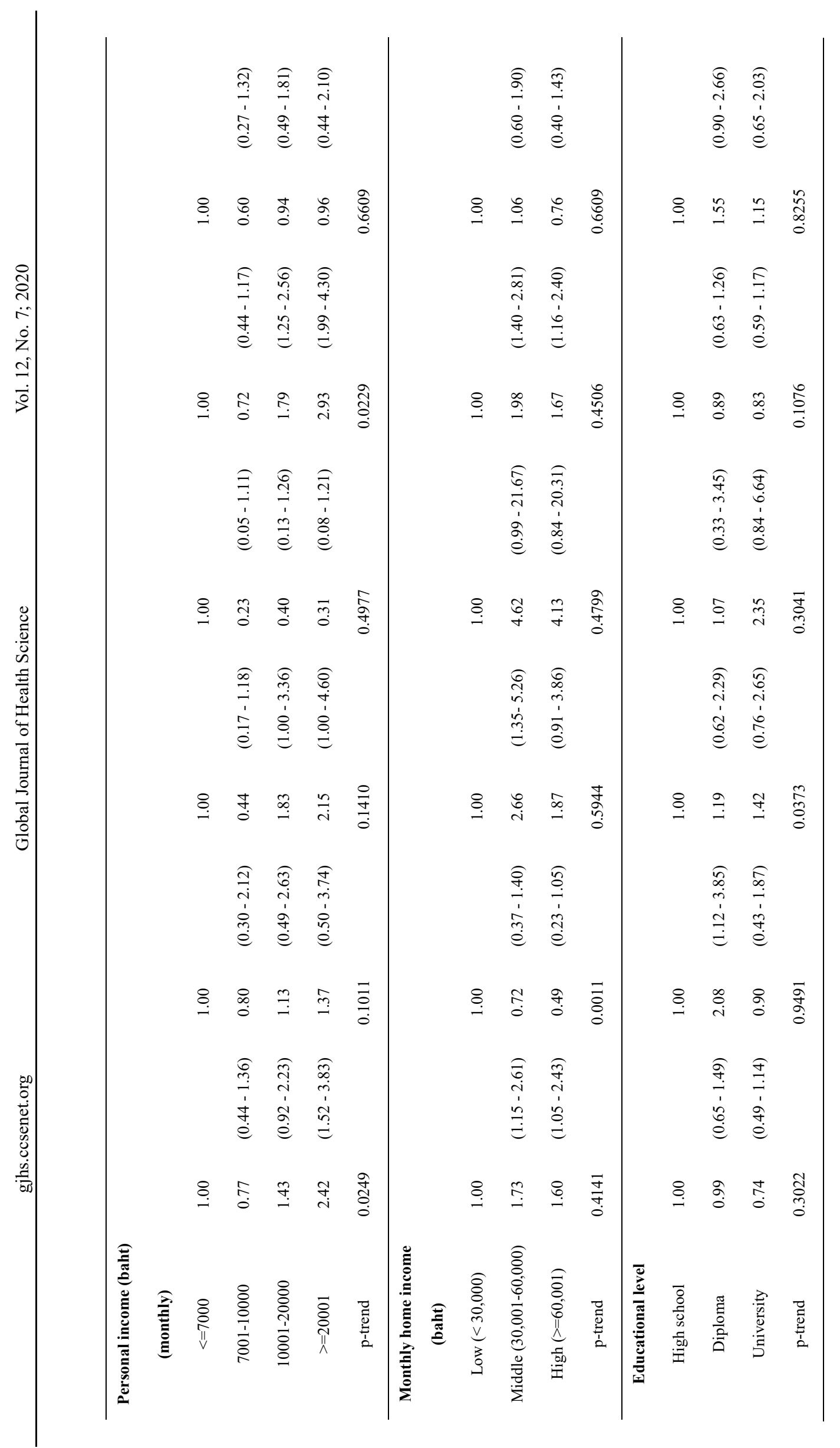




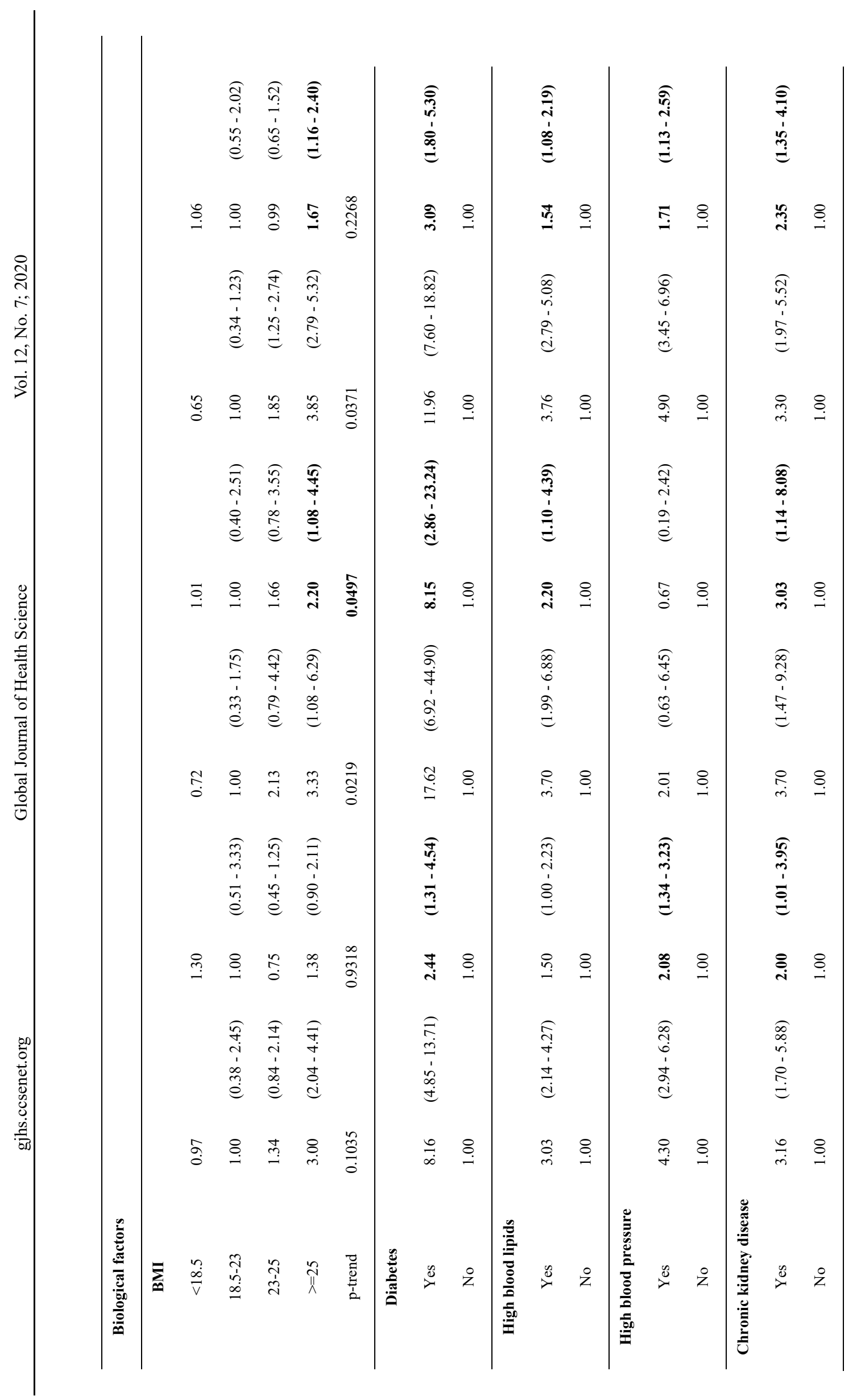




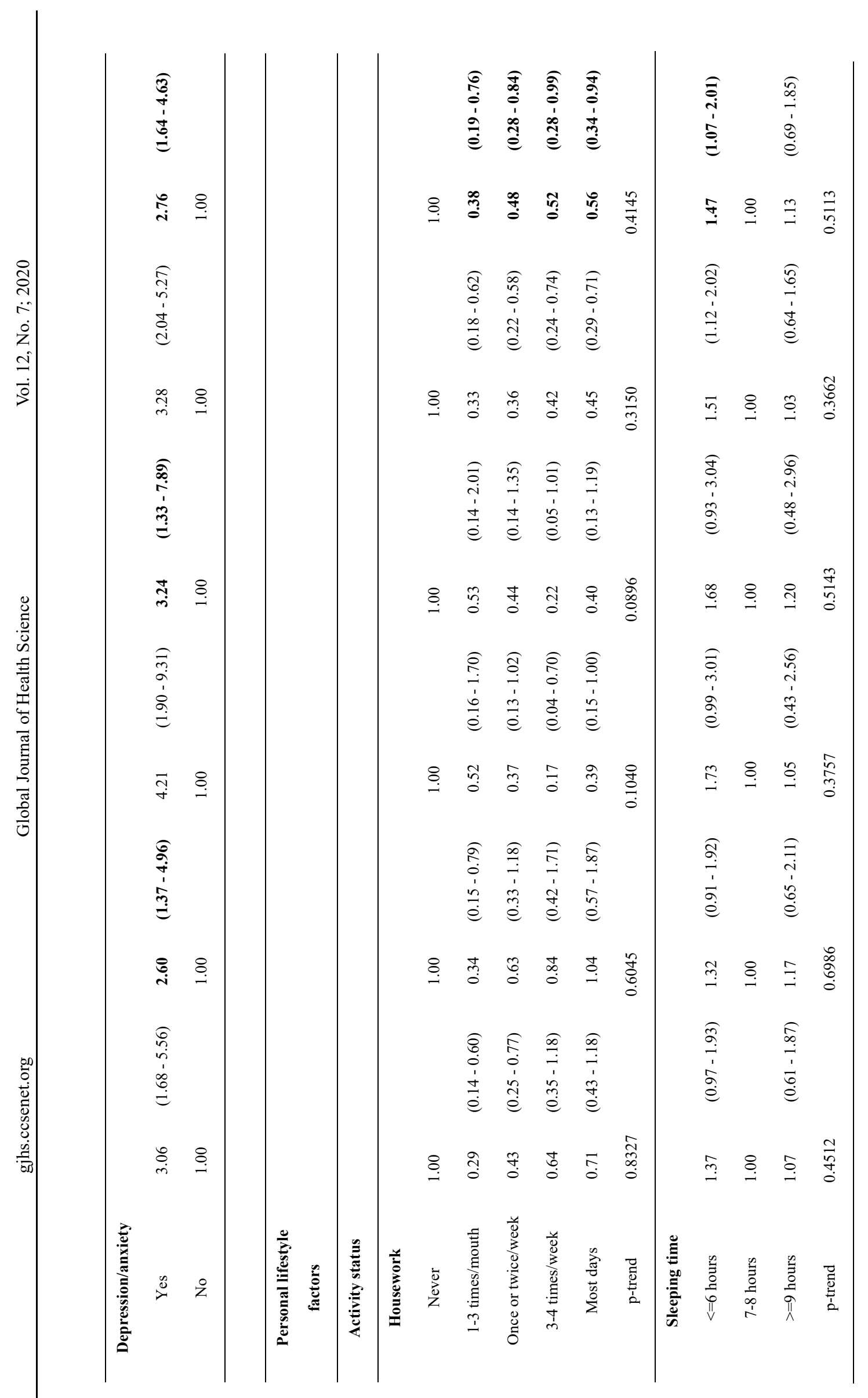




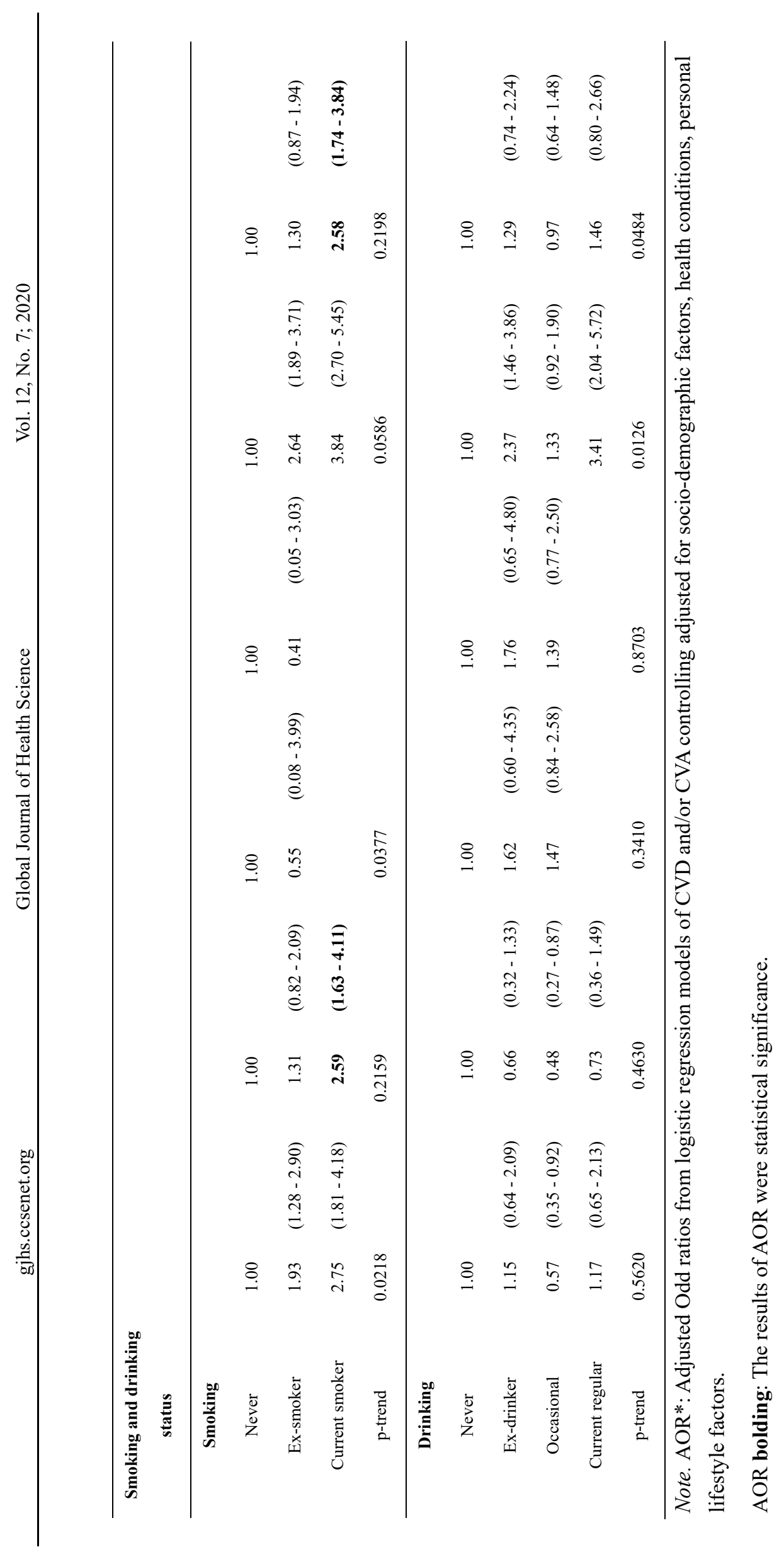


Table 4. The Abbreviations

\begin{tabular}{cc}
\hline Cardiovascular disease & CVD \\
\hline Cerebrovascular disease & CVA \\
\hline The World Health Organisation & WHO \\
\hline Disability-Adjusted Life Year & SES \\
\hline Socio-economic status & NCDs \\
\hline Non-communicable chronic diseases & TCS \\
\hline Thai cohort study & STOU \\
\hline Sukhothai Thammathirat Open University & RR \\
\hline Rural-Rural & RU \\
\hline Rural-Urban & UR \\
\hline Urban-Rural & UU \\
\hline Urban-Urban & BMI \\
\hline Body mass index & $95 \%$ CI \\
\hline $95 \%$ Confidence interval & AOR \\
\hline Adjusted odds ratio &
\end{tabular}

\section{Copyrights}

Copyright for this article is retained by the author(s), with first publication rights granted to the journal.

This is an open-access article distributed under the terms and conditions of the Creative Commons Attribution license (http://creativecommons.org/licenses/by/4.0/). 